\title{
Quality factors of nonideal micro pillars
}

\section{Gregersen, Niels; Nielsen, Torben Roland; Tromborg, Bjarne; Mørk, Jesper}

\section{Published in:}

Applied Physics Letters

Link to article, DOI:

$10.1063 / 1.2751586$

Publication date:

2007

Document Version

Publisher's PDF, also known as Version of record

Link back to DTU Orbit

\section{Citation (APA):}

Gregersen, N., Nielsen, T. R., Tromborg, B., \& Mørk, J. (2007). Quality factors of nonideal micro pillars. Applied Physics Letters, 91(1), 011116. https://doi.org/10.1063/1.2751586

\section{General rights}

Copyright and moral rights for the publications made accessible in the public portal are retained by the authors and/or other copyright owners and it is a condition of accessing publications that users recognise and abide by the legal requirements associated with these rights.

- Users may download and print one copy of any publication from the public portal for the purpose of private study or research.

- You may not further distribute the material or use it for any profit-making activity or commercial gain

- You may freely distribute the URL identifying the publication in the public portal

If you believe that this document breaches copyright please contact us providing details, and we will remove access to the work immediately and investigate your claim 


\title{
Quality factors of nonideal micro pillars
}

\author{
N. Gregersen, ${ }^{a)}$ T. R. Nielsen, B. Tromborg, and J. Mørk \\ COM.DTU, Department of Communications, Optics and Materials, Nano.DTU, Technical University \\ of Denmark, Building 343, DK-2800 Kongens Lyngby, Denmark
}

(Received 13 April 2007; accepted 30 May 2007; published online 6 July 2007)

\begin{abstract}
The influence of fabrication-induced imperfections and material absorption on the quality $(Q)$ factor of a microcavity pillar is studied numerically. The dependence on sidewall inclination, selective underetch, and intrinsic loss is quantified. The authors show that imperfections can lead to an improvement in $Q$ and that a sidewall inclination angle of less than $1^{\circ}$ causes a dramatic change in the $Q$ factor. The variations in $Q$ can be attributed to a delicate balance between effective index contrasts, mode overlap, and higher-order mode contributions. (C) 2007 American Institute of Physics. [DOI: 10.1063/1.2751586]
\end{abstract}

In an optical microcavity, the spontaneous emission rate of an atom can be modified compared to its value in free space by modifying the mode structure of the electromagnetic field in which the atom is embedded. ${ }^{1,2}$ The spontaneous emission rate in an optical geometry under study divided by that in a bulk material is known as the Purcell factor. ${ }^{2}$ It is proportional to the cavity quality $(Q)$ factor over the effective mode volume and can be increased or reduced by tailoring the optical modes. In a high- $Q$ cavity, where the photon lifetime is long compared to the field-atom interaction time, coherent coupling can be achieved ${ }^{3}$ leading to a reversible energy exchange between the field and the embedded emitter and entanglement of the corresponding states.

A solid-state system suitable for studying these effects is the semiconductor micro pillar (MP). It consists of a cavity surrounded by two distributed Bragg reflectors (DBRs), where confinement in the lateral plane is assured by total internal reflection. A single quantum dot (QD) placed inside the cavity allows the MP to be used as a single-photon source. The highly reflecting DBRs and the cavity result in a high- $Q$ value and a low mode volume is obtained by choosing a small pillar diameter. Light is predominantly emitted through the top DBR in a mode profile suitable for coupling to an optical fiber.

Strong coupling between the QD and the cavity mode has recently been demonstrated ${ }^{4}$ in a MP, and emission of single indistinguishable photons from MPs was reported in Ref. 5. In both references, the performance depends critically on the $Q$ factor of the cavity, and for this reason the investigation of $Q$ factors in MPs as function of geometry has recently been initiated. Optimization rules for MP designs have been investigated, ${ }^{6}$ and the dependence of $Q$ on pillar diameter has been analyzed theoretically using a formulation based on Bloch modes. ${ }^{7,8}$ A recent paper ${ }^{9}$ compares experimentally measured and numerically computed $Q$ factors, where a detailed model taking into account selective AlAs underetch has been introduced, and an increase in $Q$ for a particular etch depth is observed, but not explained. We extend these studies by investigating the influence of the sidewall inclination apparent in Fig. 1. of Ref. 9 and we identify the physical mechanism behind the variation in $Q$ for the

\footnotetext{
${ }^{a)}$ Electronic mail: ngr@com.dtu.dk
}

selective AlAs underetch. Also, the role of intrinsic absorption by the host material is investigated.

We use the eigenmode expansion technique ${ }^{10}$ (EET) to study the $Q$ factors. The EET allows a full three-dimensional vectorial solution of the wave equation, necessary when considering MPs of small diameters and in general whenever diffraction effects are dominating. In this frequency domain technique, the MP structure is divided into layers of uniform refractive index profile along a propagation axis, usually chosen as the $z$ axis. In each layer, eigenmodes are determined for the particular index profile assuming uniformity along the entire $z$ axis. The optical field in each layer is expanded on the corresponding eigenmodes, and the fields at each side of the interface between adjacent layers are connected using the scattering matrix formalism. ${ }^{11}$

The $Q$ factor is usually ${ }^{7-9}$ evaluated by determining a complex pole of a scattering matrix. However, a complex wavelength in the EET results in a substantial performance penalty in the determination of eigenvalues, so instead we calculate the $Q$ factor in the following way. Light propagating through the cavity is partially reflected at the top and bottom DBR mirrors and the sum of this infinite series of reflections is given by the total roundtrip operator,

$$
M=\left(1-R^{t} \Theta R^{b} \Theta\right)^{-1},
$$

where $R^{t, b}$ are matrices describing reflection at the top and bottom DBR mirrors respectively, ${ }^{11}$ while $\Theta$ is the translation operator propagating the modes from one end of the cavity to the other. The transmission operator of the entire MP is defined by (a)

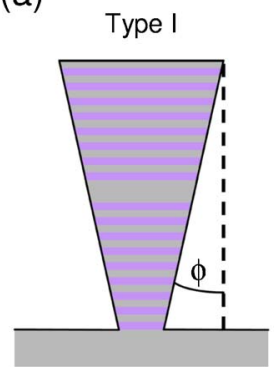

(b)

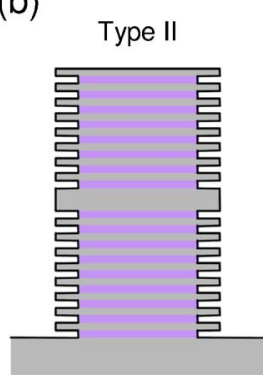

(c)

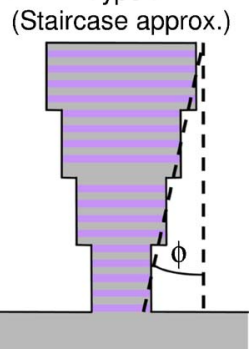

FIG. 1. (Color online) [(a) and (b)] Illustrations of the two pillar geometry imperfections considered and (c) the staircase approximation used for the type I imperfection. 

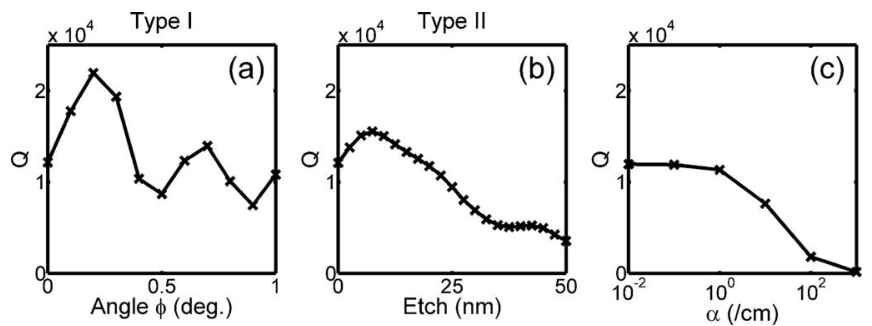

FIG. 2. $Q$ factor for (a) type I and (b) type II imperfections and (c) under influence of material loss.

$$
T=T^{b} \Theta M T^{t},
$$

with $T^{t, b}$ describing transmission through the top and bottom DBRs, respectively. Since the quantities $T^{t, b}$ and $\Theta$ vary slowly compared to the roundtrip operator $M$, a resonance in $M$ corresponds to a resonance in the transmitted light spectrum. The quantity $|\operatorname{det}(M)|^{2}$ is then calculated as function of wavelength, a resonance peak is identified and the center position and the full width at half maximum are extracted to obtain the $Q$ factor.

We consider a GaAs/AlAs MP with 26 and $29 \frac{1}{2}$ layer pairs in the top and bottom DBR mirrors using typical AlGaAs parameters. The GaAs (AlAs) layer thickness is 72 (79) $\mathrm{nm}$, the cavity thickness is $294 \mathrm{~nm}$, the pillar diameter for the ideal geometry, i.e., without imperfections, is $1 \mu \mathrm{m}$, and wavelength-independent refractive indices of 3.542 (2.958) for GaAs (AlAs) are used. The structure, which is similar to those considered in Refs. 12 and 13, has a primary resonance wavelength of $\sim 969 \mathrm{~nm}$ and the $Q$ factor is $\sim 12000$. The $Q$ of the corresponding planar structure with infinite pillar diameter is $\sim 187000$.

Two types of geometries (Fig. 1) mimicking fabricationinduced imperfections are investigated. For type I, the MP has a conical shape corresponding to the effect of underetch from reactive ion etching. For type II, the AlAs layers have a smaller diameter than the GaAs layers, simulating the selective oxidation-assisted underetch of the AlAs layers from the sidewalls reported in Ref. 14. We also consider the effect of a uniform intrinsic material loss $\alpha$ on the ideal MP. The geometry for type I imperfection is modeled using the staircase approximation sketched in Fig. 1(c), where the number of staircase steps is increased until convergence is obtained. For angles up to $1^{\circ}, 32$ staircase steps were required.

The $Q$ factor as function of degree of geometry imperfection and material loss is shown in Fig. 2. For type I imperfection, an increase in $Q$ is observed with a peak at an
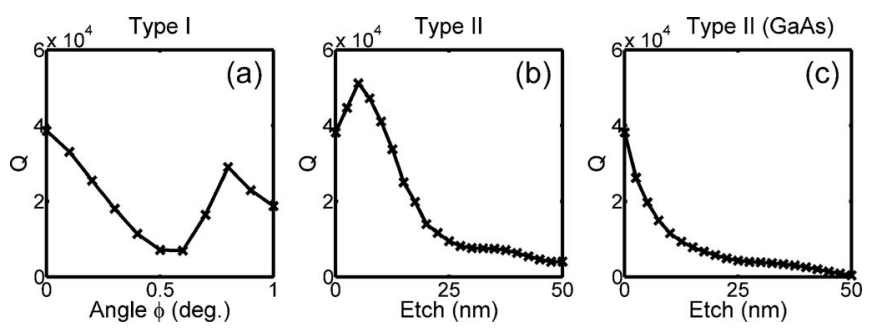

FIG. 3. $Q$ factor for the optimized structure, in (a) for type I imperfection, in (b) for type II imperfection, and in (c) for type II imperfection with an artificial GaAs etch.

inclination angle of $0.2^{\circ}$ followed by a minor peak at $0.7^{\circ}$, and we note that within a variation in angle of just $1^{\circ}$, the $Q$ factor changes in magnitude by almost a factor of 3 . The $Q$ factor also increases for type II imperfection with a peak for an AlAs underetch of $\sim 10 \mathrm{~nm}$. These increases in $Q$ are somewhat counterintuitive as one would expect imperfections to have a negative influence on the $Q$ factor. In Fig. 2(c), the effect of intrinsic loss on the $Q$ factor is plotted, and we estimate that the effective absorption coefficient should be larger than $\sim 20 \mathrm{~cm}^{-1}$ to result in a reduction of the $Q$ factor of more than a factor of 2. Since the material losses of GaAs/AlAs at the relevant wavelengths for a passive structure are much smaller ${ }^{15}$ than $20 \mathrm{~cm}^{-1}$, intrinsic loss is not an important influence on the $Q$ factor.

To identify the origin of the increases in the $Q$ factor for increasing sidewall inclination and etch depth, we point out that the MP layer thicknesses are close to those of experimentally given structures ${ }^{12}$ where the layer thickness is typically chosen on the basis of a one-dimensional planar-cavity transfer matrix calculation. Thus, for a given pillar diameter the layers are not optimized in order to maximize the DBR reflectivity for the fundamental mode. In what follows, we determine the $Q$ factors as a function of geometry imperfections in an optimized MP geometry. The layer thicknesses of this structure are determined by calculating the effective index $n_{\text {eff }}$ of the fundamental $\mathrm{HE}_{11}$ mode and by setting the layers thicknesses equal to $\lambda /\left(4 n_{\text {eff }}\right)$ and the cavity thickness equal to $\lambda / n_{\text {eff }}$. In our simulations, the design wavelength $\lambda$ was $950 \mathrm{~nm}$. The $Q$ factor as a function of geometry imperfection for this optimized geometry is illustrated in Fig. 3.

In the optimized structure, ${ }^{16}$ the peak at $0.2^{\circ}$ for type I imperfection has disappeared, and the appearance of this peak in Fig. 2(a) is due to the inclination angle producing a perturbation of the DBR mirror reflectivity towards its optimum value. However, the second peak, now shifted to $0.8^{\circ}$,
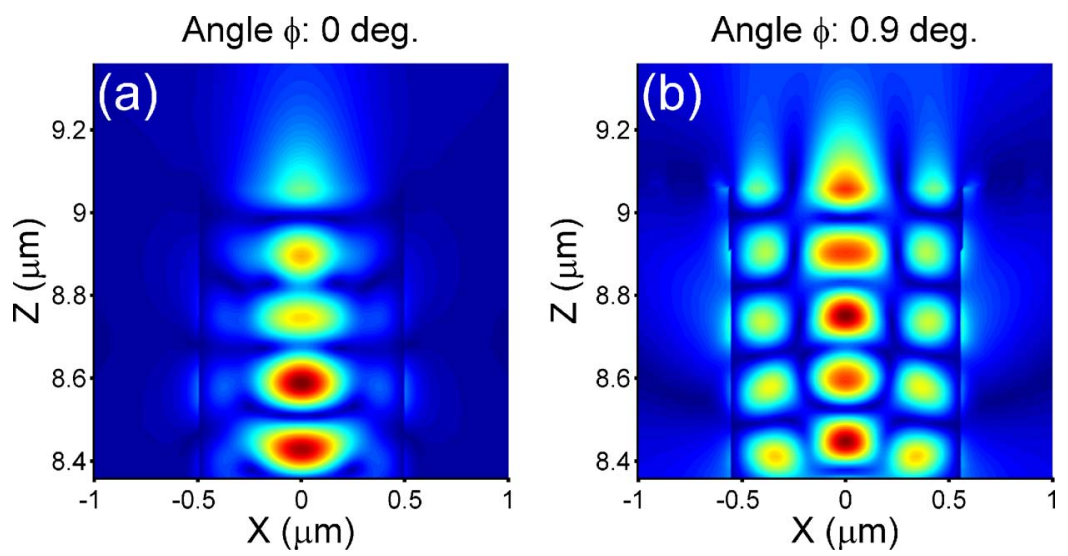

FIG. 4. (Color online) Radial electric field profiles in top DBR mirror for inclination angles $\phi$ of (a) $0^{\circ}$ and (b) $0.9^{\circ}$. 

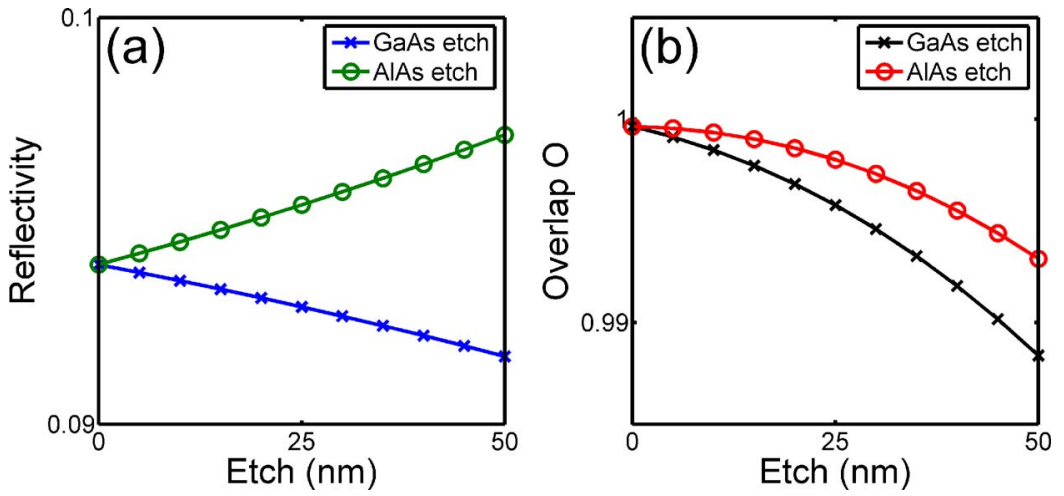

FIG. 5. (Color online) (a) Reflectivity and (b) overlap of $\mathrm{HE}_{11}$ modes in $\mathrm{GaAs}$ and $\mathrm{AlAs}$ layers as a function of etch depth. is still present. The origin of this peak can be studied by examining the electric field profile in the top of the MP. The field profile for the ideal structure depicted in Fig. 4(a) shows a fundamental mode propagating through the top DBR layers. However, the field profile for the MP with an inclination angle shown in Fig. 4(b) reveals coupling to higher order modes. Thus, when the inclination angle is increased, interaction between the $\mathrm{HE}_{11}$ mode and higher order modes assists in maintaining high Bragg mirror reflectivity. A similar higher order mode interaction, responsible for strong variations in the $Q$ factor, was also identified in Refs. 7 and 8 . However, due to lack of periodicity of the MP layers in our geometry, an analysis based on the Bloch mode formalism introduced in those papers cannot be applied here. We note that by changing the inclination angle from $0.6^{\circ}$ to $0.8^{\circ}$, the $Q$ increases by more than a factor of 4 , and the influence of the inclination angle on $Q$ is thus even more dramatic for the optimized structure.

In the case of type II imperfection, the peak near an etch of $10 \mathrm{~nm}$ is only shifted slightly in the optimized structure, as illustrated in Fig. 3(b), and the initial increase of $Q$ with AlAs underetch is still present. To study the origin of this increase, we have, in addition, evaluated the $Q$ factor as a function of an artificial underetch into the GaAs layers. This type of underetch is not observed experimentally, but we introduce it to study the physical mechanism behind the increase in $Q$. For the GaAs underetch, we observe a monotonic reduction in $Q$ in Fig. 3(c) with increasing etch and no peak is present. This behavior can be understood by considering the influence of the etch depth on the reflectivity $\left(n_{\text {eff,GaAs }}-n_{\text {eff,AlAs }}\right) /\left(n_{\text {eff,GaAs }}+n_{\text {eff,AlAs }}\right)$ at a layer interface illustrated in Fig. 5(a). When an AlAs layer diameter is reduced, the mode is pushed out of the pillar and the effective index drops towards unity. The effective index contrast between the GaAs and AlAs layers becomes larger and the DBR reflectivity is thus improved, resulting in an increase in $Q$. Similarly, the effective index of the GaAs layer becomes smaller when the diameter is decreased; however, in this case the index contrast is reduced and the DBR mirror reflectivity is only lowered. In this way, underetching of the AlAs (GaAs) layers causes an improvement (reduction) in $Q$. However, in an analysis of the DBR reflectivity, the overlap integral of the transverse components of the $\mathrm{EH}_{11}$ modes in GaAs and AlAs layers, given by

$$
O=\left|\int \mathbf{E}_{\perp, \mathrm{GaAs}} \cdot \mathbf{E}_{\perp, \mathrm{AlAs}} d \mathbf{r}_{\perp}\right|,
$$

should also be considered. Figure 5(b) illustrates the overlap as a function of etch into the GaAs or AlAs layers. In both cases the mode overlap deteriorates with decreasing layer diameter, at one point the effect of the poor overlap dominates over the effect of increased reflectivity for AlAs underetch and the $Q$ then drops from its peak value. We note that the value of $Q$ at its peak for type II imperfection is $\sim 25 \%$ higher than that of the ideal structure. Experimental control of the AlAs sidewall underetch would thus allow an increase in $Q$ and would be benificial for realizing high- $Q$ MPs.

In summary, our calculations provide estimates of the fabrication tolerance requirements for $Q$ factors of MPs. We have shown that both types of imperfections considered can lead to an improvement in $Q$ in an unoptimized MP. Also, we demonstrate that a change in inclination angle of less than $1^{\circ}$ can cause drastic variations in $Q$ and that a minor AlAs underetch from the sidewalls actually improves the $Q$ factor.

The work was supported by the EU IST project "QPhoton" (Contract No. IST-29283) and the authors thank S. Reitzenstein and A. Löffler for fruitful discussions. The authors also acknowledge access to the Sun HPC systems at the Technical University of Denmark, made possible by a collaboration with the Scientific Computing Group at IMM-DTU under Grant No. DCSC HDW-1104-08.

${ }^{1}$ E. Yablonovitch, Phys. Rev. Lett. 58, 2059 (1987).

${ }^{2}$ E. M. Purcell, Phys. Rev. 69, 681 (1946).

${ }^{3}$ K. J. Vahala, Nature (London) 424, 839 (2003).

${ }^{4}$ J. P. Reithmaier, G. Sęk, A. Löffler, C. Hofmann, S. Kuhn, S. Reitzenstein, L. V. Keldysh, V. D. Kulakovskii, T. L. Reinecke, and A. Forchel, Nature (London) 432, 197 (2004).

${ }^{5}$ I. Robert, E. Moreau, J.-M. Gérard, and I. Abram, J. Lumin. 94-95, 797 (2001).

${ }^{6}$ J. Vučković, M. Pelton, A. Scherer, and Y. Yamamoto, Phys. Rev. A 66, 023808 (2002).

${ }^{7}$ P. Lalanne, J. P. Hugonin, and J.-M. Gérard, Appl. Phys. Lett. 84, 4726 (2004).

${ }^{8}$ G. Lecamp, P. Lalanne, J. P. Hugonin, and J.-M. Gérard, IEEE J. Quantum Electron. 41, 1323 (2005).

${ }^{9}$ G. Lecamp, J. P. Hugonin, and P. Lalanne, Appl. Phys. Lett. 90, 091120 (2007).

${ }^{10}$ P. Bienstman, H. Derudder, R. Baets, F. Olyslager, and D. D. Zutter, IEEE Trans. Microwave Theory Tech. 49, 349 (2001).

${ }^{11}$ L. Li, J. Opt. Soc. Am. A 13, 1024 (1996).

${ }^{12}$ A. Löffler, J. P. Reithmaier, G. Sęk, C. Hofmann, S. Reitzenstein, M. Kamp, and A. Forchel, Appl. Phys. Lett. 86, 111105 (2005).

${ }^{13}$ J.-M. Gérard, B. Gayral, and E. Moreau, e-print arXiv:quant-ph/0207115.

${ }^{14}$ S. Varoutsis, S. Laurent, I. Sagnes, A. Lemaître, L. Ferlazzo, C. Mériadec, G. Patriarche, I. Robert-Philip, and I. Abram, J. Vac. Sci. Technol. B 26, 2499 (2005)

${ }^{15}$ Properties of Aluminium Gallium Arsenide, edited by S. Adachi (Institution of Electrical Engineers, London, 1993), pp. 126-140.

${ }^{16}$ For the optimized cavity, the diameter of the cavity center was fixed to $1.0 \mu \mathrm{m}$ for all sidewall inclinations. 